\title{
Nerve Transfer in Delayed Obstetrical Palsy Repair
}

\author{
Filippo Sénès ${ }^{1}$ Nunzio Catena ${ }^{1}$ Jacopo Sénès ${ }^{1}$ \\ ${ }^{1}$ Microsurgery and Hand Surgery Unit, Istituto Giannina Gaslini, \\ Genova, Italy \\ J Brachial Plex Peripher Nerve Inj 2015;10:e2-e14.
}

\begin{abstract}
Address for correspondence Filippo Sénès, MD, Microsurgery and Hand Surgery Unit, Istituto Giannina Gaslini, Via G. Gaslini 5, 16147 Genova, Italy (e-mail: filipposenes@ospedale-gaslini.ge.it).
\end{abstract}

\begin{abstract}
Keywords

- nerve transfer

- neurotization

- obstetrical brachial plexus palsy

- delayed nerve repair

Objective When root avulsions are detected in children suffering from obstetrical brachial plexus palsy (OBPP), neurotization procedures of different nerve trunks are commonly applied in primary brachial plexus repair, to connect distally the nerves of the upper limbs using healthy nerve structures. This article aims to outline our experience of neurotization procedures in OBPP, which involves nerve transfers in the event of delayed repair, when a primary repair has not occurred or has failed. In addition, we propose the opportunity for late repair, focusing on extending the time limit for nerve surgery beyond that which is usually recommended. Although, according to different authors, the time limit is still unclear, it is generally estimated that nerve repair should take place within the first months of life. In fact, microsurgical repair of OBPP is the technique of choice for young children with the condition who would otherwise have an unfavorable outcome. However, in certain cases the recovery process is not clearly defined so not all the patients are direct candidates for primary nerve surgery.

Methods In the period spanning January 2005 through January 2011, among a group of 105 patients suffering from OBPP, ranging from 1 month to 7 years of age, the authors have identified a group of 32 partially recovered patients. All these patients underwent selective neurotization surgery, which was performed in a period ranging from 5 months to 6.6 years of age.

Results Late neurotization of muscular groups achieved considerable functional recovery in these patients, who presented with reduced motor function during early childhood. The said patients, with the exception of five, would initially have avoided surgery because they had not met the criteria for nerve surgery.

Conclusion We have concluded that the execution of late nerve surgical procedures can be effective in children affected by OBPP.
\end{abstract}

\section{Introduction}

Even though early microsurgical repair of obstetrical brachial plexus injuries is the technique of choice for affected children who are otherwise destined to an unfavorable motor and sensory outcome, making a decision for surgery at the third or the fourth month of life, commonly considered as the most suitable time for brachial plexus exploration, is sometimes difficult. In fact, many patients presenting with incomplete

received

May 16, 2014

accepted after revision

February 19, 2015

published online

April 29, 2015 recovery of movement of palsied arm do not exactly meet the criteria for early nerve surgery, so that they need a different surgical strategy. Moreover, other patients undergo medical evaluation later than the first months of life, by which point a brachial plexus repair may have an uncertain outcome and also risks jeopardizing already achieved functions.

Nowadays, a new approach has stemmed from neurotization techniques, which are applied in primary nerve surgery
Copyright @ 2015 Georg Thieme Verlag KG Stuttgart · New York
License terms

(@) (1) $\circledast$ 
Table 1 Types of delayed neurotization and number of procedures

\begin{tabular}{|l|l|}
\hline Types of neurotization & $\begin{array}{l}\text { Number of } \\
\text { procedures }\end{array}$ \\
\hline XI to suprascapular nerve & 22 \\
\hline XI to axillary nerve & 1 \\
\hline XI to musculocutaneus nerve & 1 \\
\hline $\begin{array}{l}\text { Branch of ulnar nerve to } \\
\text { musculocutaneus nerve }\end{array}$ & 5 \\
\hline Branch of ulnar nerve to radial nerve & 4 \\
\hline Contralateral C7 root to axillary nerve & 1 \\
\hline $\begin{array}{l}\text { Lateral branch of thoracodorsal } \\
\text { nerve to long thoracic nerve }\end{array}$ & 4 \\
\hline Branch of median nerve to ulnar nerve & 1 \\
\hline Intercostal nerves to musculocutaneus nerve & 2 \\
\hline Intercostal nerves to radial nerve & 5 \\
\hline Intercostal nerves + XI to radial nerve & 1 \\
\hline Total & 47 \\
\hline
\end{tabular}

in the event of brachial plexus palsy repair in both adults and children. ${ }^{1}$

In particular the transfer of healthy nerves, which are directly connected into the terminal nerves of some muscular groups, can restore motion of the palsied arm when it is too late for safe primary nerve surgery of the brachial plexus.

Neurotization of single muscles (i.e., deltoid, external rotators of the shoulder, triceps, serratus anterior, etc.) can be obtained using fascicles or some branches of donor nerves, with the intent of not losing the donator nerve function completely.

One aim of this paper is to outline our experience of late neurotization procedures in obstetrical brachial plexus palsy (OBPP) repair.

Because the number of patients is limited, it should be kept in mind that the paper is intended to demonstrate the feasibility of nerve procedures performed in children during their first years of life, rather than to validate the results of specific techniques of neurotization.

Second, we explore extending the time limit for nerve surgery beyond that which is usually indicated in many reports in the literature. However, according to different authors, the time limit is still unclear, but it is estimated that nerve repair should take place within the first year of life and ideally between the third and the ninth months of life. ${ }^{2,3}$

Moreover, we propose some technical indications for delayed repair of obstetrical brachial plexus injuries with a view to restoring many different muscle groups.

\section{Material and Methods}

In the period spanning January 2005 through January 2011, among a group of 105 patients suffering from OBPP, ranging from 1 month to 7 years of age, we identified a group of 32 partially recovered patients, in whom the recovery process was lacking in functional motion of different muscles. Out of 105 patients suffering from OBPP, 56 were involved in upper plexus (53.3\%), 25 were presenting with intermediate palsy (23.8\%), whereas 24 had a complete brachial plexus involvement (22.9\%).

Some of these patients underwent selective neurotization surgery directed at the nerve trunks in a period ranging from 5 months to 6.6 years of age.

Among this subset of 32 patients, 15 presented with upper palsy, whereas 12 were suffering from intermediate palsy and 5 had total palsy involvement.

Forty-seven neurotization procedures were performed in the selected 32 patients (-Table $\mathbf{1}$ ).

The injured nerve trunks were revitalized by means of different donor nerves.

Before the late nerve surgery, all the patients had previously been assessed by means of clinical evaluation and neurophysiologic tests to exclude a complete denervation of the muscle, regardless of whether they had previously been surgically treated or not.

In fact, 5 patients out of the 32 had previously undergone a primary brachial plexus repair (-Table $\mathbf{2}$ ).

Table 2 Five patients underwent primary nerve surgery without a satisfactory outcome prior to delayed neurotization procedure

\begin{tabular}{|l|l|l|l|}
\hline & Type of paralysis & Primary nerve reconstruction & $\begin{array}{l}\text { Waiting period } \\
\text { for delayed nerve } \\
\text { surgery (in months) }\end{array}$ \\
\hline Case 1 & C5-C6-C7 & $\begin{array}{l}\text { Resection of upper and medium trunk neuromas-tubulization } \\
\text { of upper trunk and direct suture of medium trunk }\end{array}$ & 20 \\
\hline Case 2 & C5-C6 & $\begin{array}{l}\text { Resection of upper trunk neuroma and reconstruction by means } \\
\text { of autologous sural nerve grafts }\end{array}$ & 41 \\
\hline Case 3 & Total & $\begin{array}{l}\text { Resection of upper, medium, and inferior trunk neuromas and } \\
\text { reconstruction using 3 nerve tubes }\end{array}$ & 32 \\
\hline Case 4 & C5-C6-C7 & $\begin{array}{l}\text { Resection of upper trunk neuroma and reconstruction by means } \\
\text { of autologous sural nerve grafts and neurolysis of medium trunk }\end{array}$ & 52 \\
\hline Case 5 & Total & $\begin{array}{l}\text { Resection of upper and inferior trunk neuromas and recon- } \\
\text { struction using nerve tubes from C5 to upper trunk and C8 to } \\
\text { inferior trunk; neurolysis of medium trunk }\end{array}$ & 15 \\
\hline
\end{tabular}


e4 Nerve Transfer in Delayed Obstetrical Palsy Repair Sénès et al.

Table 3 Musculoskeletal procedures performed in association with neurotization

\begin{tabular}{|l|l|l|}
\hline Orthopedic procedure & Associated neurotization & Number of cases \\
\hline Subscapularis muscle release & Neurotization of suprascapular nerve & 22 \\
\hline Camitz opponensplasty & Neurotization of musculocutaneus nerve & 1 \\
\hline
\end{tabular}

Among those, one child was presenting with upper palsy, two were suffering from intermediate palsy, and the other two had a complete palsy.

Up to $32+/-15.11$ (months $+/$ - standard deviation [SD]) after the primary surgery, those five patients had not shown a promising recovery in some muscle function, especially in correlation with the recovery of elbow flexion.

Up to now, there are no specific criteria for identifying patients who should undergo late nerve transfers, so in our case studies we evaluated shoulder motion, scapular stability, flexion and extension of the elbow, and partially hand function, aiming to recover specific targets of the upper arm.

Consequently, making a decision on which patients were candidates for late surgery, on the one hand we evaluated the residual defects of motion of the upper arm and on the other hand the time elapsed from the neonatal trauma, taking into account that those children had been excluded for primary nerve surgery or were still lacking in function after surgery.

In many cases, this nerve surgery was associated with musculoskeletal surgical procedures (-Table 3 ), which involved the surgical release of tightened subscapularis muscle.

The sex of the patients and the type of palsy are also reported. We noticed a slight prevalence in males, who were particularly affected in the right arm. Furthermore, out of 32 patients, only 5 presented with sequelae of total palsy, whereas the other children were suffering from sequelae of upper or intermediate lesions. At the time of surgery, the average age was $31.3 \pm 19.4$ (months \pm SD), with a range of 73 months; the youngest patient was 5 months old and the oldest 6.6 years old ( - Table 4 ).
All the data are reported as mean and SD. The median range for continuous variables has also been calculated. Moreover, absolute and relative frequencies have been used for categorical variables. Parameters for the two groups were compared using the test for categorical variables. A $p$ value less than 0.05 was considered statistically significant. The statistical analysis was performed using SAS software.

\section{Surgical Indications}

In applying the different neurotizations, three major anatomical districts can be defined:

1. Shoulder (axillary, suprascapular, and long thoracic nerve)

2. Elbow (musculocutaneus and radial nerve)

3. Hand (ulnar nerve)

\section{Suprascapular Nerve}

In primary nerve surgery of the brachial plexus, suprascapular nerve reconstruction is achievable by means of grafting from $\mathrm{C} 5$ root or using accessory spinal nerve neurotization. If a comparison is drawn between the two methods, there is no significant disparity in the results. ${ }^{4-6}$

In the natural course and even after primary nerve surgery, in a vast number of patients affected by OBPP, shoulder stiffness precociously occurs, interfering with the functional outcome. Actually the causes of shoulder functional impairment are varied, though the muscular imbalance due to subscapularis contracture is considered the main cause of stiffness. ${ }^{7}$

Surgical treatment focusing on the subscapularis muscle can preserve passive shoulder movement and facilitate the revitalization of the external rotator muscles. The surgical lengthening

Table 4 General characteristics of the subjects evaluated $(n=32)$

\begin{tabular}{|l|l|l|l|}
\hline \multicolumn{2}{|l|}{ Gender $(n, \%)$} & $13(37.1)$ \\
\hline Female & $19(62.9)$ \\
\hline Male & $18(56.35)$ & \\
\hline Side $(n, \%)$ & $14(43.75)$ & \\
\hline Right & \multicolumn{3}{l|}{} \\
\hline Left & $31.3 \pm 19.4$ & Total \\
\hline Age at the time of surgery & 25.5 & 1 & All \\
\hline Mean \pm SD & 73 & 5 & 4 \\
\hline Median & C5-C6 & 7 & 5 \\
\hline Range & 7 & 12 & 13 \\
\hline Gender|type of paralysis & 8 & & 32 \\
\hline F & 15 & & \\
\hline M & & \\
\hline All & & \\
\hline
\end{tabular}


of subscapularis muscle tendon by an anterior approach has been proposed, but we favor the subscapularis muscle release from the scapular anterior aspect by means of a minimal surgical approach along the posterior pillar of the arm pit.

Subsequently, when it is feasible, we do prefer to spare the accessory spinal nerve in primary brachial plexus repair, with the intention of using it in a possible secondary nerve reconstruction (-Fig. 1). Suprascapular nerve should be considered as an independent entity in reconstructive nerve surgery of the obstetrical brachial plexus lesion, ${ }^{8}$ especially for the controversial results obtained after primary nerve surgery. A correct dissection of the accessory spinal nerve, sparing the residual trapezium muscle innervation, and an adequate nerve cooptation to provide a good axonal contribution are essential to defining a successful procedure. Frequently, a mismatch of the nerve fibers occurs so that the accessory spinal nerve is inserted into the center of the cut surface of suprascapular nerve.

\section{Axillary Nerve}

Deltoid muscle function mainly interferes in the range of motion and stability of the scapular girdle. Conversely, good shoulder abduction is essential for satisfactory shoulder joint motion.

When deltoid muscle recovery does not occur, axillary nerve reconstruction in association with suprascapularis nerve repair should be performed to achieve a better functional outcome. ${ }^{9}$

In our experience to date, we have only performed one axillary nerve reconstruction, using accessory spinal nerve as the donor nerve. On that occasion, the accessory spinal nerve was connected to the axillary nerve, which was dissected at Velpeau quadrilateral space, by means of sural nerve grafting. To restore the deltoid muscle function, a contralateral $\mathrm{C7}$ transfer could be a second option when simultaneous transfers are needed.

\section{Long Thoracic Nerve}

In a few cases, scapular winging was treated by neurotization of the thoracic long nerve, using the lateral branch of the thoracodorsal nerve (-Figs. 2 and $\mathbf{3}$ ).

The recovery of serratus anterior muscle function is indicated mainly in C5-C6-C7 lesions, usually causing an evident scapular winging, whereas in a $\mathrm{C} 5-\mathrm{C} 6$ lesion, the residual contribution of C7 allows for sufficient scapular motion. Moreover, long thoracic nerve repair, in association with suprascapular nerve repair, offers better results rather than isolated suprascapular nerve repair. ${ }^{10}$

The nerve repair can be obtained by means of lateral branch of thoracodorsal nerve or intercostal nerves.

We constantly used the lateral branch of the thoracodorsal nerve, but we consider that one intercostal nerve might serve

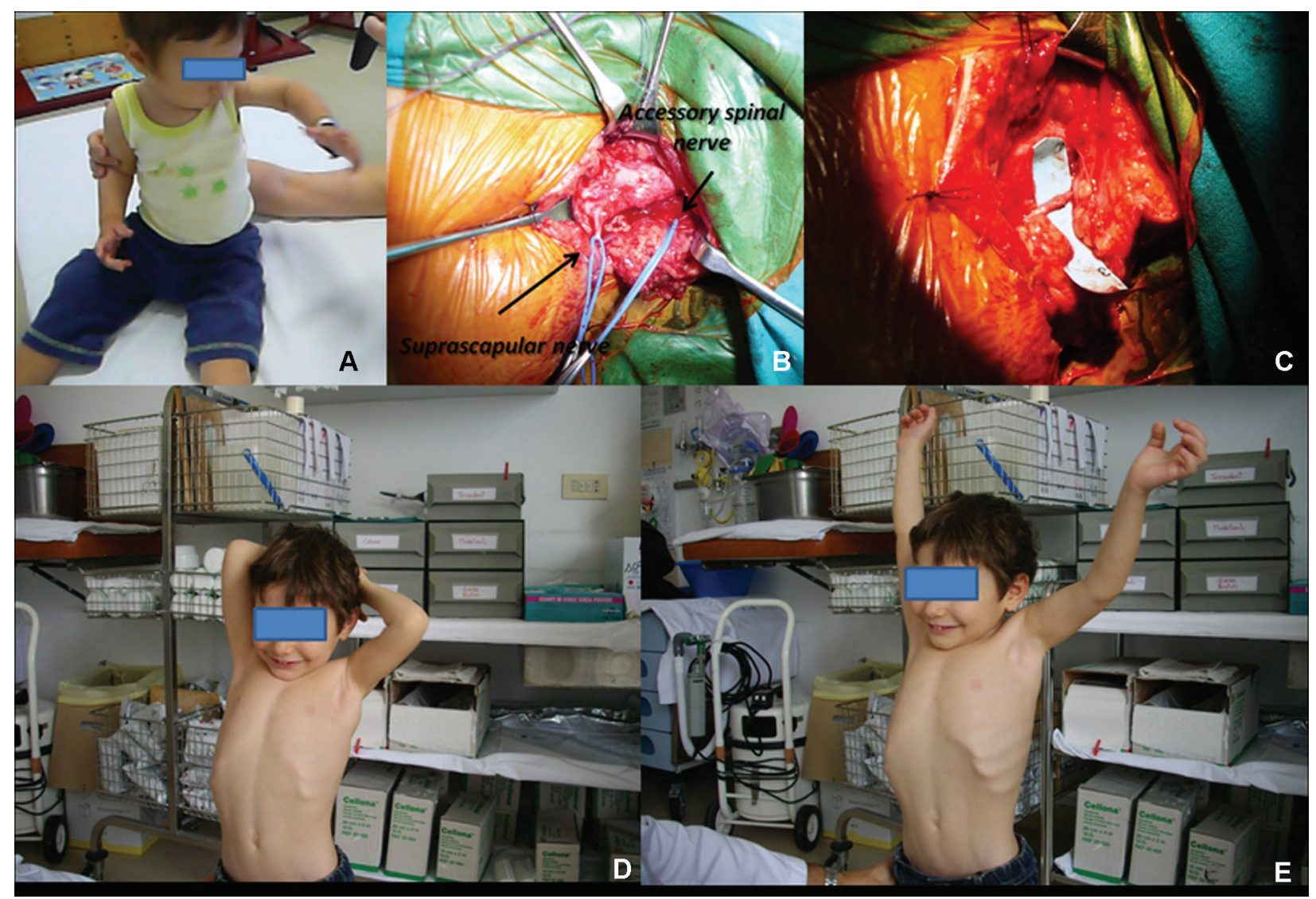

Fig. 1 Child at 1 year of age. (A) Clinical aspect demonstrating the lack of active shoulder external rotation. (B) Surgical exposure of accessory spinal nerve (XI) and suprascapular nerve (SSC). (C) XI to SSC neurotization. (D, E) Shoulder function at 4 years follow-up. 


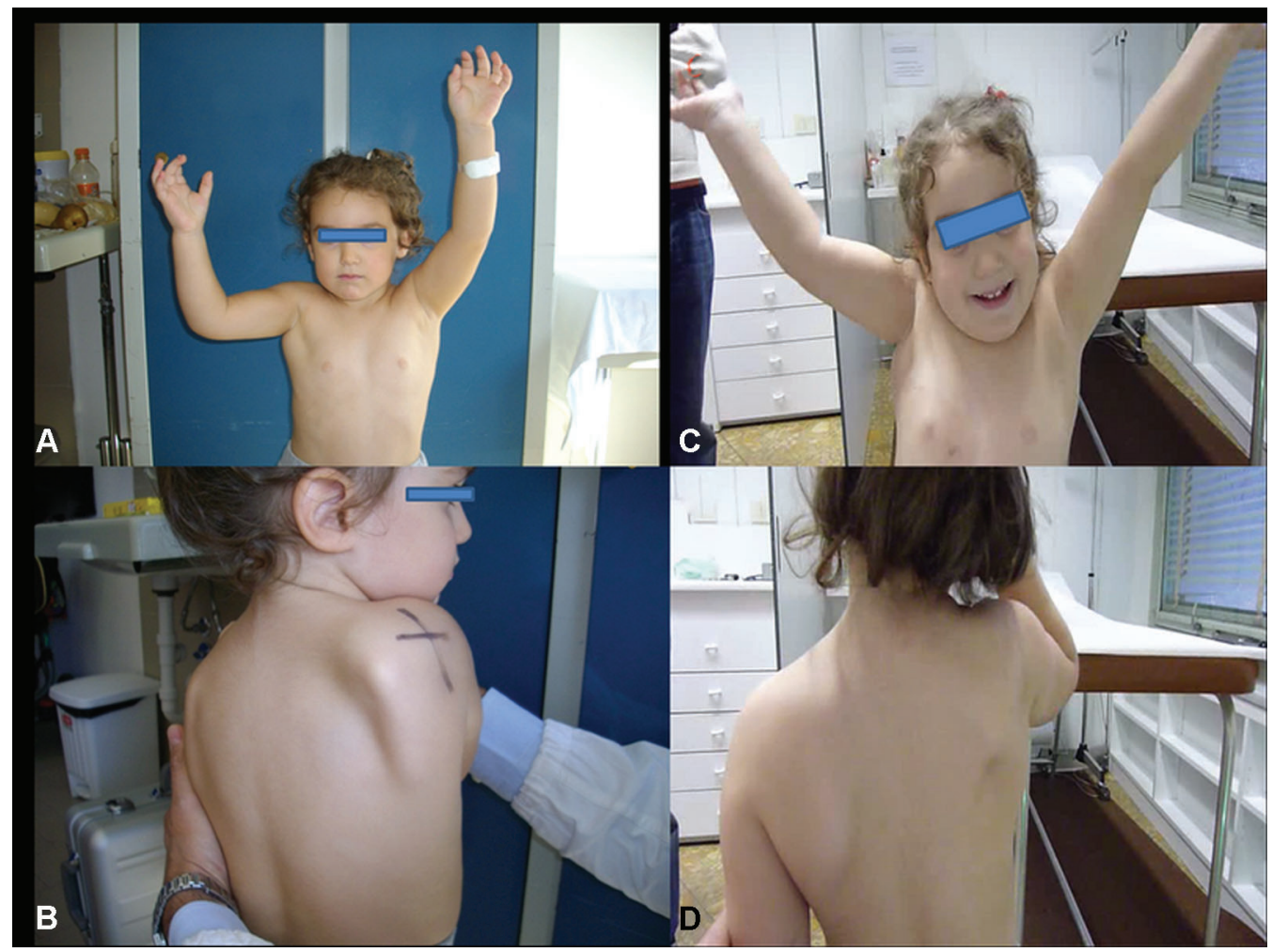

Fig. 2 Girl 3132 years old. (A, B) Before surgery the clinical appearance showed a significant winging of the scapula and reduction in shoulder abduction. (C, D) Functional outcome 1 year postoperatively.

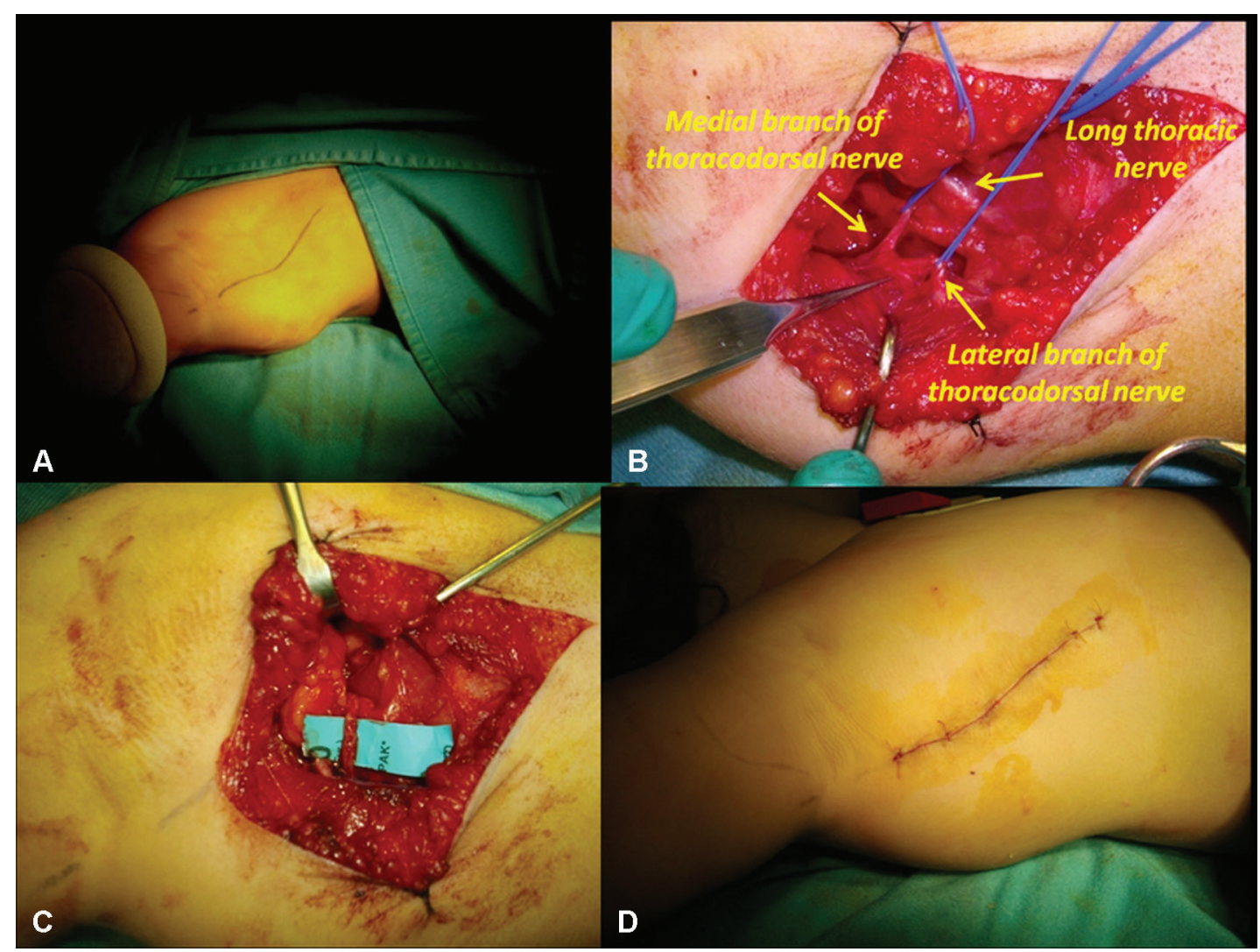

Fig. 3 Girl 31/2 years old (same patient as in previous figure): surgical procedure. (A) Skin incision in axillary region. (B) Surgical exposure of long thoracic nerve and thoracodorsal nerve (with lateral and medial branches). (C) Lateral branch of thoracodorsal nerve to repair the long thoracic nerve. (D) Appearance of the axillary region after skin suture. 
the same function, without losing power in the latissimus dorsi muscle. ${ }^{11}$

Radial nerve (branches for the long head of the triceps muscle and radial nerve trunk): The treatment of extensive obstetrical brachial plexus injury can be defined as successful if muscle recovery of the triceps is achieved. In fact, elbow extension deficit impairs normal activities during everyday life (especially when reaching for objects at a distance). Within the early years of life, patients with a lack of recovery in muscles innervated by the posterior cord of the brachial plexus, which went treated or untreated, may be in need of selective repair of deficient muscular functions. Elbow extension recovery can be obtained with the repair of two specific branches leading to the long head of the triceps muscle. In our experience, the radial nerve restoration was sometimes achieved not only by neurotization of the branches of radial nerve to the long head of triceps but also by connecting donor nerves into the radial nerve, completely resected. Some publications have illustrated satisfactory results in radial nerve repair using the intercostal nerves, despite the fact that the musculocutaneous nerve constantly demonstrates a higher level of recovery (-Fig. 4). ${ }^{12-14}$

According to the clinical evaluation, it needs to be emphasized that reconstruction was different in each single case. In addition to the transfer of three intercostal nerves connected to the radial nerve trunk, which more frequently occurred, in four patients some fascicles of the ulnar nerve were the donor nerves that were chosen for neurotizing nerve branches of the long head of the triceps muscle, as a modified Oberlin's procedure. The ulnar nerve fascicles leading to the flexor carpi ulnaris muscle were transposed into the nerve branches of the long head of the triceps muscle. Once the ulnar nerve was dissected at a proximal third of the arm, the nerve fascicles of the ulnar nerve were passed through the axillary folder to reach the branches of the long head of the triceps muscle using a posterior approach and by means of sural nerve grafting (-Figs. 5 and $\mathbf{6}$ ).

In the remaining three cases, a simultaneous exposure of the ulnar and radial nerves in the axillary folder occurred, obtaining a direct nerve suture without grafting.

In the reconstruction of the extensor function of the upper limb, we have never found any problem in using nerve trunks leading to the antagonistic muscle. Thus in OBPP, without any involvement of the lower roots, we consider the ulnar nerve fascicles to be the number one choice for delayed repair of the elbow extension. Conversely, when the lower plexus is impaired, the intercostal nerves always represent a good alternative.

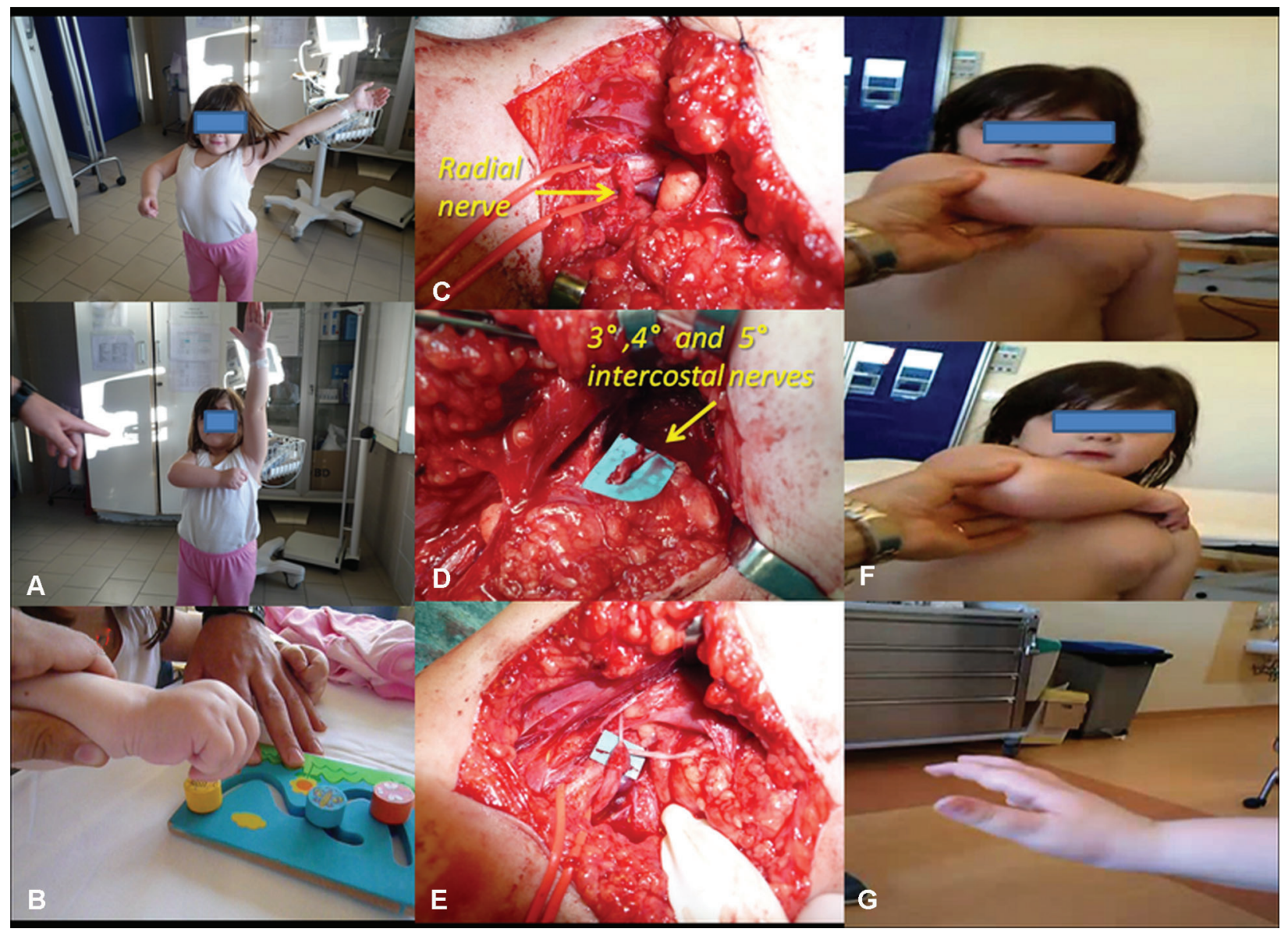

Fig. 4 Girl 31/2 years old. (A, B) Lack of active elbow extension and absence of digital extension. (C-E) surgical exposure of radial nerve (RAD) at the axillary folder and 3- and 4-degree intercostal nerves (IC) dissection. Neurotization of the IC into the RAD. (F, G) Elbow and hand functions 8 months after surgery. 


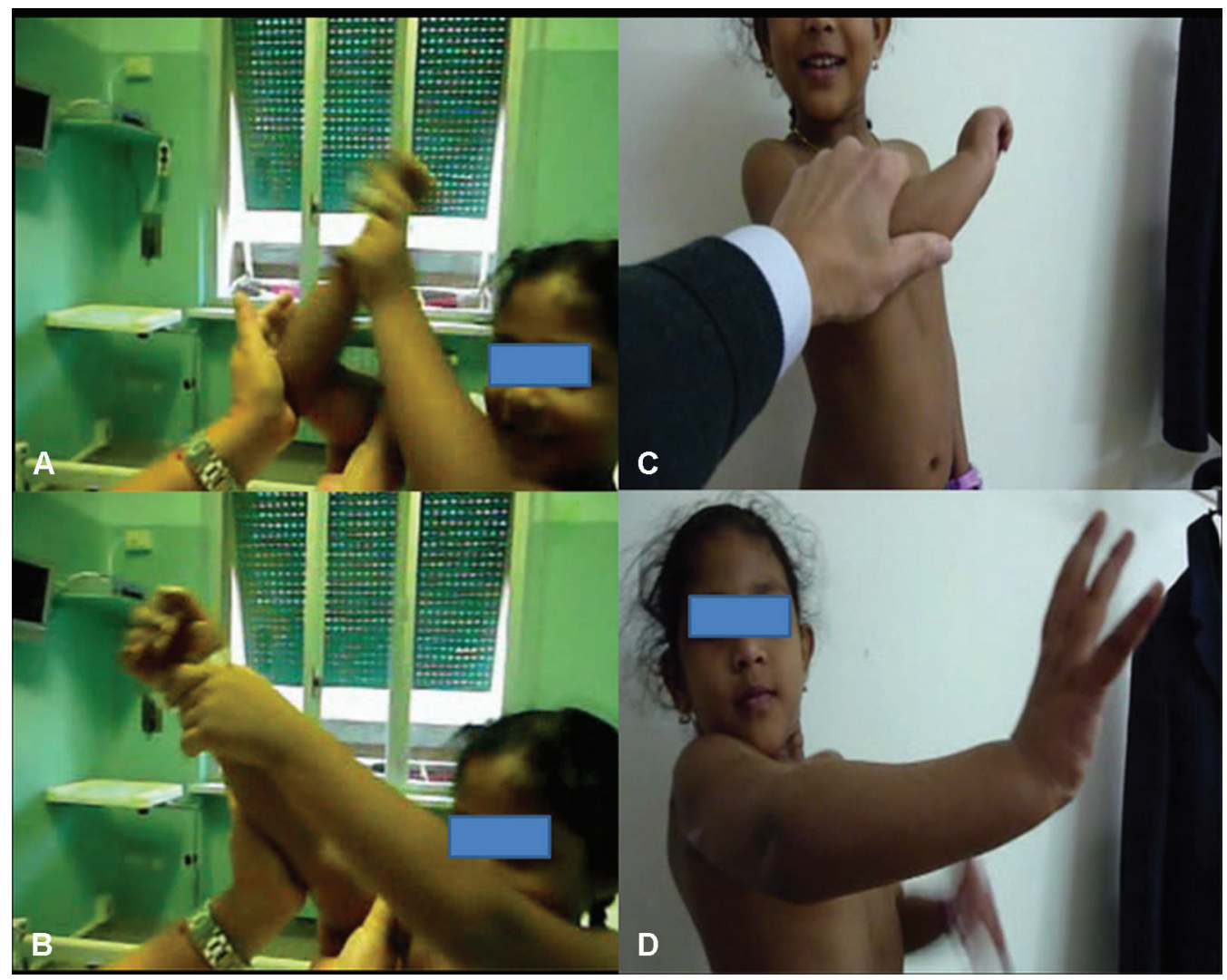

Fig. 5 Girl 41/2 years old: clinical aspect. (A, B) Before surgery the clinical aspect demonstrated a good active elbow flexion but the lack of active elbow extension, due to tricipital muscle palsy. The extension of the elbow could only be reached with the help of the opposite upper limb. (C, D) Improved elbow extension 6 months after neurotization.

\section{Musculocutaneous Nerve}

Reinnervation of the biceps brachialis muscle obtained by means of musculocutaneous neurotization is the ideal procedure to achieve active elbow flexion.

This procedure offers better results in comparison to muscle transfer for elbow flexion restoration. ${ }^{15}$ Literature related to elbow flexion recovery by way of the so-called Oberlin's procedure (a branch of ulnar nerve transferred onto the musculocutaneous nerve) or intercostal nerves transposition has often been published, but the former is possibly the most widespread procedure of neurotisation. ${ }^{16,17}$

A real advantage of ulnar nerve fascicle or intercostal nerve neurotization derived from a single direct suture without interposition grafts is that it allows for faster muscular reinnervation. In the surgical planning of nerve repair for OBPP, the ulnar nerve involvement should be identified. What is more, anatomical variations of the musculocutaneous nerve or a double-level nerve lesion through the entry point of the coracobrachialis muscle should also be considered. In our experience, elbow flexion was restored using both Oberlin's technique and the intercostal nerves. Although we more commonly directed the nerve transfer into the musculocutaneous nerve fascicles without an electrical response at the time of surgery, we sometimes neurotized the branch of the brachialis muscle in certain patients who presented with some electromyographic (EMG) activity in the biceps muscle but who showed no elbow flexion. We also successfully performed the accessory spinal nerve neurotization of the musculocutaneous nerve on one occasion. In our opinion, where applicable, the first option should be the ulnar nerve fascicle transfer because it makes for a faster procedure and less donor site damage, in addition to avoiding possible shortand long-term complications. If during the course of clinical evaluation or intraoperative electrostimulation, the ulnar nerve is deficient, restoration of elbow flexion is achieved by means of the intercostal nerves. When we availed of the intercostal nerves, we generally used three to four nerves ( $\mathrm{T} 3$, $\mathrm{T} 4, \mathrm{~T} 5$, and T6) without any complication, even in the case of younger patients. Similarly in the combined reconstruction of musculocutaneous and radial nerves, intercostal nerves are used to restore elbow flexion, whereas ulnar nerve fascicles are used for elbow extension. An additional option with a view to achieving elbow flexion might be accessory spinal nerve neurotization of the musculocutaneous nerve, but the procedure requires nerve grafting and should not be considered the first choice technique.

Median nerve as a donor is a further option, but we have no experience in using median nerve fascicles for elbow flexion recovery, as in the subset of patient in question, we did not find the indication for that late neurotisation. ${ }^{18}$

Moreover in our opinion, when dealing with extensive brachial plexus palsy in which the ulnar nerve is impaired, the reliability of the median nerve for elbow flexion recovery might be poor. 


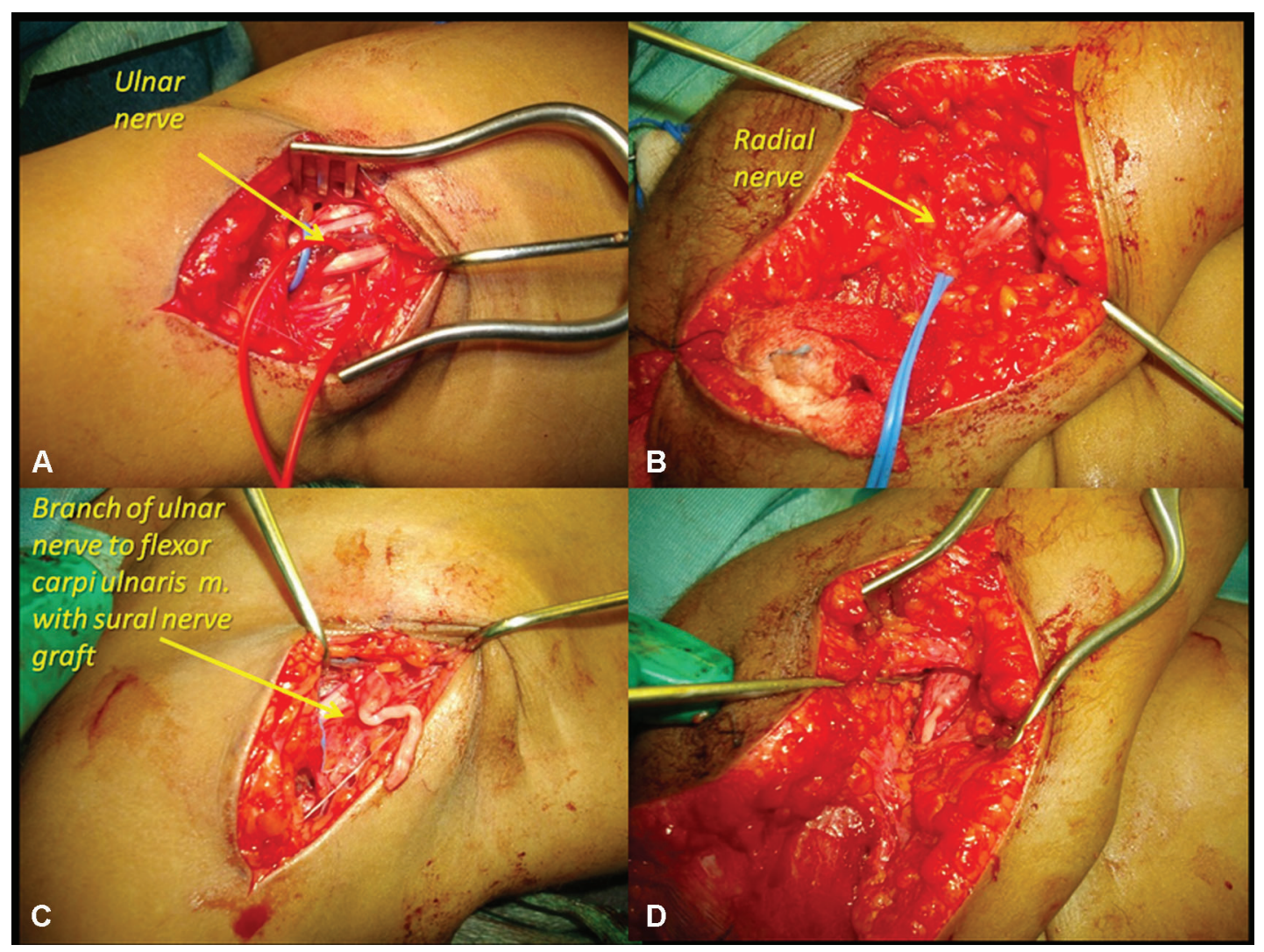

Fig. 6 Girl 41/2 years old (same patient as in previous figure): surgical procedure. (A) Exposure of the ulnar nerve at the axillary folder. (B) Microsurgical dissection of the branches leading to the flexor carpi ulnaris muscle. (C) Exposure of the radial nerve at the proximal region of the arm. (D) Ulnar nerve branches transposed into radial nerve by means of sural nerve grafts interposition.

In multiple nerve transfers for severe brachial plexus palsy sequelae, contralateral $\mathrm{C} 7$ to restore the muscles function might be a further option. ${ }^{19,20}$

\section{Hand}

Selective neurotizations are generally indicated for motor recovery of the proximal segment of the upper arm when adequate sensation in the distal segment is represented.

With regard to intrinsic muscle recovery, satisfactory hand function is a difficult target to reach even after primary nerve surgery.

In 1999, Battiston and Lanzetta proposed a double distal neurotization in traumatic high ulnar nerve injury, using the median nerve as a donor. ${ }^{21}$

In subtotal OBPP with partial recovery of lower roots, a spontaneous recovery that is often incomplete sometimes occurs. Consequently, some function of the median nerve might appear. In those patients, we propose a hypothetical distal neurotization from the median nerve to revitalize the ulnar nerve fascicles leading to the interosseous muscles. To date, we have only experienced this technique on one occasion and we were quite satisfied with the outcome.

\section{Results}

The mean follow-up was $27.5 \pm 18.2$ (months \pm SD). Regarding some patients, the follow-up period might be considered short, but we aimed to outline the possibility of using peripheral nerve repair after OBPP, regardless of the final outcome. On the other hand, the majority of patients showed promising functional results in a rather short time after surgery.

The results were evaluated using several scores for different anatomical regions:

- Shoulder: Mallet score. ${ }^{22}$

- Scapular stability: the winging of the shoulder blade was evaluated on a 3-degree scale ( $\mathrm{I}=$ minimal to no winging, varying from 15 to 45 degrees; II = partial winging, varying from 45 to 60 degrees, and III $=$ complete winging of the scapula, varying from 60 to 90 degrees).

- Elbow: scale of Nerve Injury Committee of British Medical Research modified by Mackinnon and Dellon. ${ }^{23}$

- Hand: Gilbert-Raimondi score. ${ }^{24}$

The results are reported identifying (a) the anatomical site of muscle failure, (b) the type of neurotization applied, and (c) the quality of the recovery.

Because in our series a vast number of patients tended precociously to develop shoulder stiffness, external rotator muscles in the shoulder were reinnervated using the accessory spinal nerve to neurotize the suprascapular nerve. This was done both as isolated procedure or associated with other selective neurotizations. Of 22 procedures that we performed, we observed 14 good, 5 fair, and 3 bad results. In 

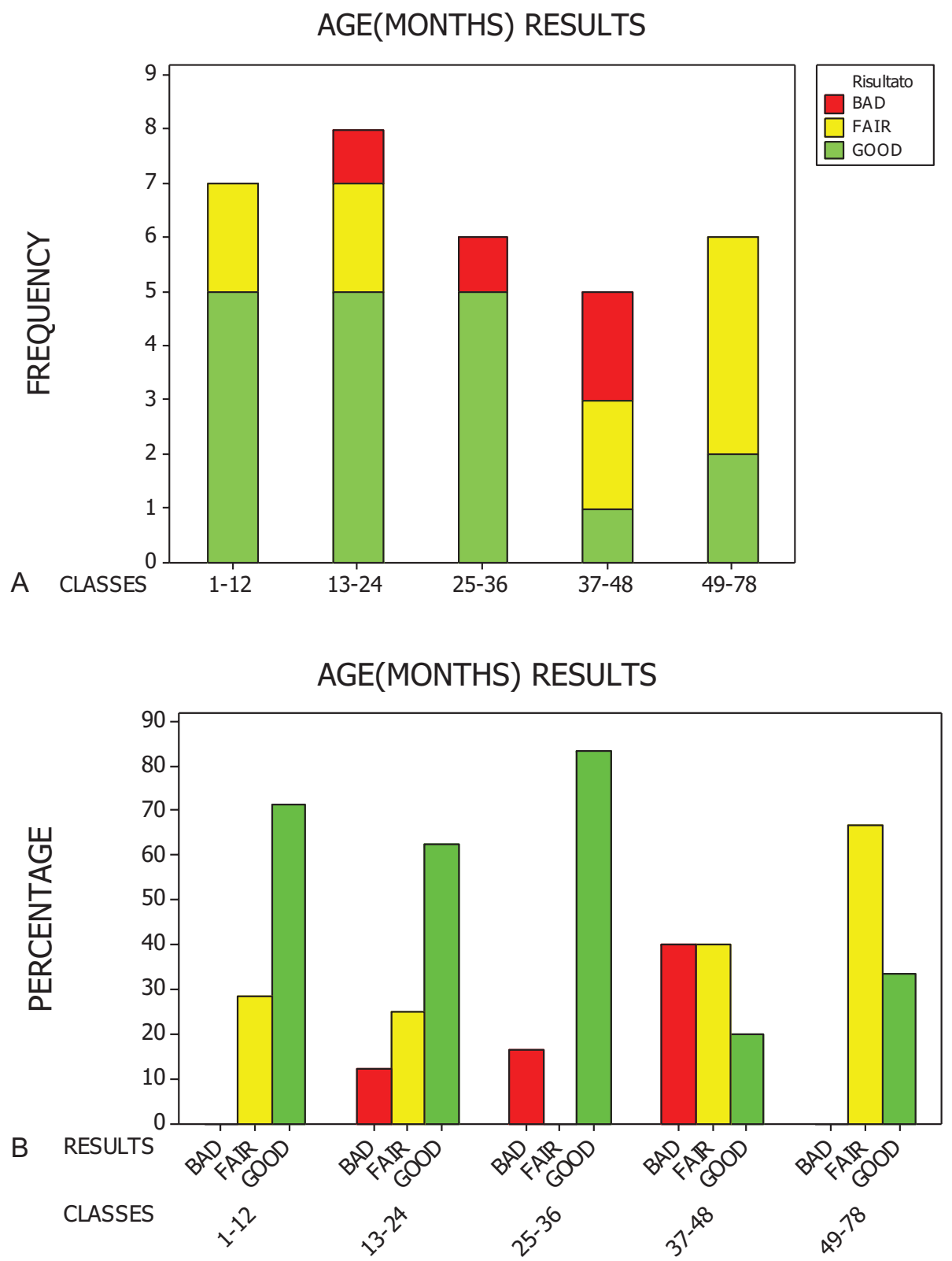

Fig. 7 (A) On the vertical axis, the table shows the results (good, fair, bad) divided into age groups, which is expressed by their age in terms of months. (B) On the horizontal axis, the table shows the results (good, fair, bad) subdivided by age group, which is expressed by their age in terms of months. The percentages are given for each group of cases. The sum of the percentages for each group is 100 . You may note that as the child's age increases, no increment is witnessed in the event of negative results. Moreover, unsatisfactory results are completely absent in the group ranging from 49 to 78 months.

our opinion suprascapularis nerve neurotization confirms the reliability of the surgical technique, having obtained $63 \%$ of positive results (Mallet IV) in patients who underwent the procedure. After consequent checkups on our case studies regarding selective neurotizations of the shoulder, an additional observation is the decreasing of muscular transfers (i.e., latissimus dorsi muscle transfer), as opposed to what happened in the past before adopting the delayed neurotization procedure. $^{25}$

In another group of four patients who underwent long thoracic nerve neurotization to achieve shoulder stabiliza- tion, reduced scapular winging, and improved internal rotation of the shoulder, we had three good results and one fair outcome.

Axillary nerve reconstruction, using the accessory spinal nerve as a donor nerve, was performed once. The patient achieved a good result, whereas another patient obtained a fair result, using contralateral $\mathrm{C} 7$ to restore the deltoid function.

In repairing the radial nerve mainly to achieve elbow extension, we operated on 10 patients using the modified Oberlin procedure four times, intercostal nerve transfers five 
Table 5 General results of delayed neurotizations

\begin{tabular}{|c|c|c|c|c|}
\hline Procedure & Good & Fair & Bad & Total \\
\hline $\mathrm{XI}$ to SSC & 14 & 5 & 3 & 22 \\
\hline$X I$ to $A X$ & 1 & 0 & 0 & 1 \\
\hline C7 CROSS to AX & 0 & 1 & 0 & 1 \\
\hline Oberlin & 1 & 2 & 2 & 5 \\
\hline IC to $\mathrm{MC}$ & 1 & 1 & 0 & 2 \\
\hline $\mathrm{XI}$ to $\mathrm{MC}$ & 0 & 1 & 0 & 1 \\
\hline Oberlin to RAD & 4 & 0 & 0 & 4 \\
\hline IC to RAD & 3 & 2 & 0 & 5 \\
\hline $\mathrm{IC}+\mathrm{XI}$ to $\mathrm{RAD}$ & 1 & 0 & 0 & 1 \\
\hline TD to TL & 3 & 1 & 0 & 4 \\
\hline MED to UL & 0 & 1 & 0 & 1 \\
\hline Total & 28 & 14 & 5 & 47 \\
\hline
\end{tabular}

Abbreviations: AX, axillary nerve; C7 CROSS, contralateral C7; IC, intercostal nerves; MC, musculocutaneus nerve; MED, median nerve; Oberlin, branch of ulnar nerve to flexor carpi ulnari; RAD, radial nerve; SSC, suprascapular nerve; TD, lateral branch of thoracodorsal nerve; TL, long thoracic nerve; UL, ulnar nerve; $\mathrm{XI}$, accessory spinal nerve.

Table 6 Results of delayed neurotizations, taking single muscles into consideration

\begin{tabular}{|c|c|c|c|c|c|}
\hline Shoulder: Mallet & Procedure & Good (Mallet IV) & Fair (Mallet III) & Bad (Mallet II) & Total \\
\hline & $\mathrm{XI}$ to SSC & 14 & 5 & 3 & 22 \\
\hline & $\mathrm{XI}$ to $\mathrm{AX}$ & 1 & 0 & 0 & 1 \\
\hline & C7 CROSS to AX & 0 & 1 & 0 & 1 \\
\hline & Total & 15 & 6 & 3 & 24 \\
\hline \multirow[t]{5}{*}{ Biceps: BMC } & Procedure & Good (>M4) & Fair (M4 - /M3 + ) & Bad (<M3) & Total \\
\hline & $\mathrm{XI}$ to $\mathrm{MC}$ & 0 & 1 & 0 & 1 \\
\hline & Oberlin & 1 & 2 & 2 & 5 \\
\hline & IC to $M C$ & 1 & 1 & 0 & 2 \\
\hline & Total & 2 & 4 & 2 & 8 \\
\hline \multirow[t]{5}{*}{ Triceps: BMC } & Procedure & Good (>M4) & Fair (M4 - /M3 +) & Bad $(<\mathrm{M} 3)$ & Total \\
\hline & Oberlin to RAD & 4 & 0 & 0 & 4 \\
\hline & IC to RAD & 3 & 2 & 0 & 5 \\
\hline & $\mathrm{IC}+\mathrm{XI}$ to RAD & 1 & 0 & 0 & 1 \\
\hline & Total & 8 & 2 & 0 & 10 \\
\hline \multirow[t]{3}{*}{ Serratus: AC } & Procedure & Good (>M4) & Fair (M4 - /M3 +) & Bad $(<\mathrm{M} 3)$ & Total \\
\hline & TD to $\mathrm{TL}$ & 3 & 1 & 0 & 4 \\
\hline & Total & 3 & 1 & 0 & 4 \\
\hline \multirow[t]{3}{*}{ Intrinsic: GILB RAIM } & Procedure & Good (Hand 5-6) & Fair (Hand 3-4) & Bad (Hand 1-2) & Total \\
\hline & MED to UL & 0 & 1 & 0 & 1 \\
\hline & Total & 0 & 1 & 0 & 1 \\
\hline
\end{tabular}

Abbreviations: AC, authors' classification; AX, axillary nerve; BMC, scale of Nerve Injury Committee of British Medical Res. modified by Mackinnon and Dellon; C7 CROSS, contralateral C7; GILB RAIM, Gilbert-Raimondi hand score; IC, intercostal nerves; Mallet, Mallet score; MC, musculocutaneus nerve; MED, median nerve; Oberlin, branch of ulnar nerve to flexor carpi ulnari muscle; RAD, radial nerve; SSC, suprascapular nerve; TD, lateral branch of thoracodorsal nerve; TL, thoracic long nerve; UL, ulnar nerve; XI, accessory spinal nerve. 
times, and only once did we perform the combined transfer of intercostal and accessory spinal nerves. The checkups demonstrated good results ( $>$ M4) in 8 cases out of 10 .

Both neurolysis of brachial plexus and selective neurotization of the radial nerve were performed in the youngest child in our sample, who had presented with elbow flexion but a lack of radial nerve function. In our experience of radial nerve neurotization (10 cases treated to date), we constantly achieved satisfactory results. In our case studies, we primarily considered elbow extension recovery, with no mention of wrist and finger extension improvement, that in three cases we also noticed.

In our experience, elbow flexion was mainly restored using both Oberlin's technique and the intercostal nerves. Active elbow flexion was achieved in six patients out of eight (biceps muscle $>$ M3) with similar results using both Oberlin's procedure and intercostal nerves transfer.

A distal neurotization from the median nerve to the ulnar nerve fascicles, which were distributed to interosseous muscles, obtained a fair result.

The group of five patients who underwent primary nerve surgery of brachial plexus with poor results required a second surgical approach for the recovery of upper limb functions . The secondary surgery was performed using procedures of neurotization, although the outcome was contrasting. We would like to point out that the lack of active elbow flexion was a common point that characterized those patients. In that group, we evaluated one good, two fair, and two bad results.

In our case studies, we cannot explain the reason of poor results obtained using Oberlin's neurotization in the group of patients, who had already undergone nerve surgery of brachial plexus. A possible explanation might be the exhaustion of musculocutaneous nerve, due to the previous nerve surgery. The situation would be different for patients who underwent secondary neurotization as first surgery.

The findings are summarized in - Tables $\mathbf{5}$ and $\mathbf{6}$.

Through analyzing our case studies, an average good outcome has been reached, despite the amount of time that had elapsed since the nerve lesion. The statistical analysis has also demonstrated that the percentage of negative outcomes has not increased in the event of a child being older than the recommended age at the time of surgery. In fact, some positive outcomes have been recorded, irrespective of the child's age at the time of surgery (-Fig. 7).

\section{Discussion}

Microsurgical repair of obstetrical brachial plexus injuries is the technique of choice for affected children, who are destined to an unfavorable motor and sensory outcome. The real advantage of direct exploration of nerve trunks is to be able to assess the level of nerve damage more correctly and to perform a more accurate nerve repair.

Nowadays different strategies have been identified even in primary nerve surgery. As neurotizations are widely used, sometime when dealing with upper palsy the doubt of operating on by means of classic repair with nerve grafts or applying different nerve procedures arises.
Beyond doubt early nerve surgery should be proposed for total palsy with no signs of recovery. It follows the surgical repair course by nerve grafts or neurotization (intra- or extraplexual) similar to the one adopted in the case of brachial plexus lesions during adulthood. Depending on the amount of damaged roots, the surgeon has to decide on using external neurotization together with intraplexual repair.

With this in mind, the surgeon can choose a better strategy to reach functional recovery of the upper arm. To date, the exact timing for primary nerve surgery is still a controversial issue.

According to Gilbert-Tassin's ${ }^{26}$ indications, the lack of spontaneous recovery of the biceps brachii muscle after the third month of life is a precise indicator, highlighting the need to proceed with microsurgical revision of the injured brachial plexus. On the other hand, some authors have estimated a different evaluation period for surgery in babies, which is highly recommended within the first year of life. ${ }^{2,3}$

In addition to children who are destined to having an unfavorable outcome, which has been identified within the first months of life, there is also another group of patients, which present with doubtful signs of spontaneous recovery or of showing partial recovery of certain muscles. Provided that the decision about which patients should undergo primary nerve surgery is often difficult, a different surgical approach should be identified for patients presenting with signs of recovery, to obtain a selective repair of the deficient muscular functions. The possibility to undergo early microsurgical repair would have been ruled out in the case of the above patients.

Undoubtedly, it is mandatory to operate on children affected by total palsy and who show no sign of recovery, even if they exceed the maximum age established for primary nerve surgery.

In the past, some authors proposed to perform a late brachial plexus surgery operating on older children affected by total palsy, even though they had no hope of recovery. Their findings were not clear and the results are still unknown.

In fact, when the repair of a whole brachial plexus is lately executed, within the first few years of development, there is a greater risk of not securing effective arm function, because of the loss of the regenerative potential of the nerve. What is more, considering the functional complexity and the distribution of brachial plexus nerve trunks, the risk of failure due to performing late surgical procedures on the brachial plexus is greatly increased. Furthermore, the risk of operating on some roots using them as intraplexual donors to restore the motion of some muscles (i.e., upper roots) might cause the loss of previously recovered upper function.

Conversely, the recent trend of reinnervating single muscles during brachial plexus repair both in children and adults has incited a new possibility to obtain a selective repair of the deficient muscular functions in children who have surpassed the recommended time frame for brachial plexus repair. In our opinion, the following three groups of patients suffering loss of movement subsequent to an OBPP should be the beneficiaries of the new surgical approach: 
1. Cases of OBPP, which initially proved promising and thus evaded nerve surgery within the first months of life, because the clinical evaluation was not clearly indicative of surgery.

2. Patients with partial recovery, who were evaluated too late to have successful primary nerve surgery.

3. Patients unsuccessfully treated with prior nerve surgery early in life. In their case, selective neurotization may represent the possibility to resume and complete primary nerve surgery.

One objection deriving from the late neurotization procedure is the absence of motor function in a prolonged denervated muscle.

In reality, EMG studies may offer some ulterior indications, but it should be taken into account that in OBPP a complete denervation of the muscles rarely occurs. More often, neuroma-in-continuity allows for the recording of some neurogenic potential (i.e., late fibrillation potentials). The aforementioned is an expression of muscle vitality; nevertheless, there is no functional motion of the muscular complex. ${ }^{27,28}$

One of the main objectives of the neurotization procedure is to reach a functional result, preventing the loss of previous functions, even if those might be minimal.

Just to be clear, patients underwent nerve surgery when the motion of the single district was very poor, that is not exceeding one-fifth MRC. The whole nerve was not cut off entirely if some electrical response was obtained. This was done to avoid resetting the nerve conduction in the hope that the donor nerve could restore function

For that reason, during surgical intervention, an accurate stimulation of the nerve trunk is performed, leaving the nerve fascicles untouched, which demonstrates an electrical response that is consequently expressed in muscle contraction. On the other hand, nerve fascicles giving a minimal or an absent contraction of the muscle under electrical stimulation should be neurotized. If the mismatch of nerve fibers from the donor nerve in comparison to the fibers of the recipient nerve is taken into account, this approach permits the reinnervation of the nerve trunk without forgetting the topographic distribution of the nerve.

The most controversial issue is the timeline for late nerve transfer in repairing OBPP sequelae.

With regard to the scarcity of publications, it is not clear at which age surgery is indicated, so it is difficult to hypothesize the time limit for performing a delayed neurotization procedure. With reference to this uncertain time limit, we operated on patients up to a maximum age of 6.6 years.

In our opinion, up until 6 years of age could be deemed acceptable, if the time that has elapsed since the obstetrical lesion and the prolonged muscular denervation is taken into account. However, this does not rule out the possibility of surgery on children of an older age.

\section{Conclusion}

To sum up, we have concluded that the execution of late nerve surgical procedures is really feasible in children affected by
OBPP. In our opinion, up until the age of 6 could be deemed acceptable, taking the time that has elapsed since the obstetrical lesion and the prolonged muscular denervation into consideration. However, this does not rule out the possibility of surgery on older children. In fact, the potential for nerve regeneration or nerve trunk recovery allows for delayed surgical intervention. Selective neurotization of muscular groups proved to achieve considerable functional recovery for those patients who presented with partial recovery of motion during early childhood. Until a few years ago, those patients would have been treated with orthopaedic secondary surgery, often with unsatisfactory results and with functional loss over the years. To avoid confusion, it should be emphasized that delayed neurotization procedure is a supplementary surgical method to help the patients attain a better outcome, precisely when primary nerve reconstruction is no longer viable. Accordingly, the neurotization procedure should not be considered an alternative procedure to brachial plexus reconstruction, which achieves the best result in the treatment of OBPP.

\section{References}

1 El-Gammal TA, El-Sayed A, Kotb MM, Saleh WR, Ragheb YF, el-Refai $O$. Delayed selective neurotization for restoration of elbow and hand functions in late presenting obstetrical brachial plexus palsy. J Reconstr Microsurg 2014;30(4):271-274

2 Michelow BJ, Clarke HM, Curtis CG, Zuker RM, Seifu Y, Andrews DF. The natural history of obstetrical brachial plexus palsy. Plast Reconstr Surg 1994;93(4):675-680, discussion 681

3 Waters PM. Obstetric brachial plexus injuries: evaluation and management. J Am Acad Orthop Surg 1997;5(4):205-214

4 Tse R, Marcus JR, Curtis CG, Dupuis A, Clarke HM. Suprascapular nerve reconstruction in obstetrical brachial plexus palsy: spinal accessory nerve transfer versus C5 root grafting. Plast Reconstr Surg 2011;127(6):2391-2396

5 van Ouwerkerk WJ, Uitdehaag BM, Strijers RL, et al. Accessory nerve to suprascapular nerve transfer to restore shoulder exorotation in otherwise spontaneously recovered obstetric brachial plexus lesions. Neurosurgery 2006;59(4):858-867, discussion 867-869

6 Pondaag W, de Boer R, van Wijlen-Hempel MS, Hofstede-Buitenhuis SM, Malessy MJ. External rotation as a result of suprascapular nerve neurotization in obstetric brachial plexus lesions. Neurosurgery 2005;57(3):530-537, discussion 530-537

7 Waters PM. Comparison of the natural history, the outcome of microsurgical repair, and the outcome of operative reconstruction in brachial plexus birth palsy. J Bone Joint Surg Am 1999;81(5): 649-659

8 Terzis JK, Kostas I. Outcomes with suprascapular nerve reconstruction in obstetrical brachial plexus patients. Plast Reconstr Surg 2008;121(4):1267-1278

9 Terzis JK, Kokkalis ZT. Shoulder function following primary axillary nerve reconstruction in obstetrical brachial plexus patients. Plast Reconstr Surg 2008;122(5):1457-1469

10 Suzuki K, Doi K, Hattori Y, Pagsaligan JM. Long-term results of spinal accessory nerve transfer to the suprascapular nerve in upper-type paralysis of brachial plexus injury. J Reconstr Microsurg 2007;23(6):295-299

11 Yamada T, Doi K, Hattori Y, Hoshino S, Sakamoto S, Arakawa Y. Long thoracic nerve neurotization for restoration of shoulder function in C5-7 brachial plexus preganglionic injuries: case report. J Hand Surg Am 2010;35(9):1427-1431 
12 Doi K, Sakai K, Kuwata N, Ihara K, Kawai S. Reconstruction of finger and elbow function after complete avulsion of the brachial plexus. J Hand Surg Am 1991;16(5):796-803

13 Terzis JK, Kokkalis ZT. Restoration of elbow extension after primary reconstruction in obstetric brachial plexus palsy. J Pediatr Orthop 2010;30(2):161-168

14 Goubier JN, Teboul F, Khalifa H. Reanimation of elbow extension with intercostal nerves transfers in total brachial plexus palsies. Microsurgery 2011;31(1):7-11

15 Noaman HH, Shiha AE, Bahm J. Oberlin's ulnar nerve transfer to the biceps motor nerve in obstetric brachial plexus palsy: indications, and good and bad results. Microsurgery 2004;24(3):182-187

16 Oberlin C, Béal D, Leechavengvongs S, Salon A, Dauge MC, Sarcy JJ. Nerve transfer to biceps muscle using a part of ulnar nerve for C5C6 avulsion of the brachial plexus: anatomical study and report of four cases. J Hand Surg Am 1994;19(2):232-237

17 Kawabata H, Shibata T, Matsui Y, Yasui N. Use of intercostal nerves for neurotization of the musculocutaneous nerve in infants with birthrelated brachial plexus palsy. J Neurosurg 2001;94(3):386-391

18 Little KJ, Zlotolow DA, Soldado F, Cornwall R, Kozin SH. Early functional recovery of elbow flexion and supination following median and/or ulnar nerve fascicle transfer in upper neonatal brachial plexus palsy. J Bone Joint Surg Am 2014;96(3):215-221

19 Lin H, Hou C, Chen D. Contralateral C7 transfer for the treatment of upper obstetrical brachial plexus palsy. Pediatr Surg Int 2011; 27(9):997-1001
20 Lin H, Hou C, Chen D. Modified C7 neurotization for the treatment of obstetrical brachial plexus palsy. Muscle Nerve 2010;42(5): 764-768

21 Battiston B, Lanzetta M. Reconstruction of high ulnar nerve lesions by distal double median to ulnar nerve transfer. J Hand Surg Am 1999;24(6):1185-1191

22 Mallet J. Paralysie obstetricale. Rev Chir Orthop Reparatrice Appar Mot 1972;58(Suppl 1):166-168

23 Mackinnon SE, Dellon AL. Surgery of the Peripheral Nerve. New York, NY: Thieme; 1988

24 Raimondi P. Evaluation of results in obstetrical brachial plexus palsy: the hand. In: Proceedings of the International Meeting of Obstetrical Brachial Plexus Palsy; Heerlen, The Netherlands; 1993

25 Pagnotta A, Haerle M, Gilbert A. Long-term results on abduction and external rotation of the shoulder after latissimus dorsi transfer for sequelae of obstetric palsy. Clin Orthop Relat Res 2004;(426): 199-205

26 Gilbert A, Tassin JL. Surgical repair of the brachial plexus in obstetric paralysis [in French]. Chirurgie 1984;110(1):70-75

27 El-Gammal TA, Abdel-Latif MM, Kotb MM, et al. Intercostal nerve transfer in infants with obstetric brachial plexus palsy. Microsurgery 2008;28(7):499-504

28 Raimbault J. Electrical assessment of muscle denervation. In: Terzis J, ed. Microreconstruction of Nerve Injuries. Philadelphia, PA: WB Saunders; 1987:211-221 